\title{
Chromosome research scholarly communication prize 2010
}

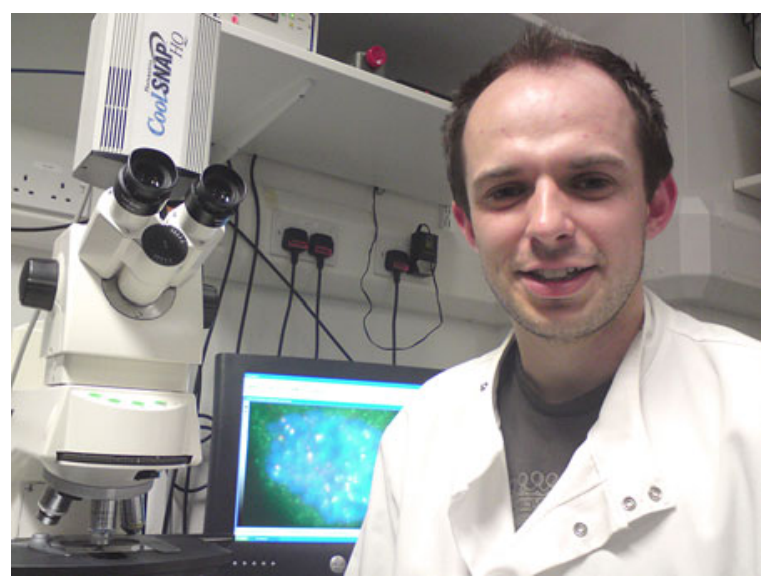

At the EMBO Workshop on Chromosome Segregation and Aneuploidy in Edinburgh (Scotland) in June 2010, our publisher, Springer SBM, generously agreed to offer a Chromosome Research Scholarly Communication Prize for the best talk given by a post-graduate student or post-doctoral researcher.

The prize was awarded to

\section{Fred Westhorpe}

Wellcome Trust Ph.D. Student in the Laboratory for Molecular Cancer Studies, Faculty of Life Sciences University of Manchester, UK.

Fred's current research activity, which formed the basis of his talk at Edinburgh, was focussed on the failure of chromosomes to segregate equally.

A key stage of mitosis is the stable attachment of all chromosomes to spindle microtubules and their alignment at the equator during prometaphase. When all chromosomes have aligned, a cell has reached metaphase. The "search and capture" of chromosomes by spindle microtubules during prometaphase is stochastic in nature and, therefore, takes individual cells differing amounts of time to complete. However, the next stage of mitosis, anaphase, is a global event: All chromosomes divide as one into sister chromatids that are pulled to the individual daughter cells. Cells therefore require a control mechanism that delays the onset of anaphase until metaphase has been achieved. This is the function of the spindle assembly checkpoint (SAC).

The SAC detects signals from unattached kinetochores, large multimeric protein structures situated at the centromeres. Fred is investigating a poorly understood area of SAC signalling: "How is the SAC itself regulated to only signal from unattached kinetochores?" and "How is the SAC turned off upon satisfaction, when all kinetochores are stably attached?" 